\title{
Effects of Dexmedetomidine Infusions on Hemodynamic Stability in Patients undergoing Laparoscopic Cholecystectomy
}

\author{
${ }^{1}$ Himanshu Dodeja, ${ }^{2}$ Vinaya Udaybhaskar, ${ }^{3}$ Amol Singam
}

\begin{abstract}
Objectives: In laparoscopic cholecystectomy, there has been emphasis on maintaining hemodynamic stability by avoiding hypertension, hypotension, or tachycardia. The hemodynamic instability is persistent during the duration of pneumoperitoneum (PNP), namely, $\mathrm{CO}_{2}$ insufflations. This study helps us to find out the efficacy of dexmedetomidine on cardiovascular system stability in patients undergoing laparoscopic cholecystectomy.
\end{abstract}

Materials and methods: Thirty patients were randomized into two groups of 15 members each: group P (placebo group) and group $D$ (dexmedetomidine group). In the former, patients received $0.9 \% 20 \mathrm{~mL}$ normal saline, while in the latter, patients received $0.4 \mu \mathrm{g} / \mathrm{kg} / \mathrm{hr}$ of injection dexmedetomidine in $0.9 \%$ normal saline. In all patients, age, weight, height, systolic blood pressure (SBP), diastolic blood pressure (DBP), and heart rate $(\mathrm{HR})$ were recorded.

Results: Dexmedetomidine being a highly selective and potent and specific alpha 2 agonist attenuates the hemodynamic response to tracheal intubation, decreases plasma catecholamine concentration during anesthesia, and decreases perioperative requirements of inhaled anesthetics. We found that dexmedetomidine helped in blunting the pressor response during the intubation, kept the HR and mean arterial pressure stable intraoperatively, and there was faster recovery as the requirement of inhalation agent was decreased intraoperatively.

Conclusion: The drug dexmedetomidine maintained cardiovascular stability during laparoscopic cholecystectomy. The inhalational agent (isoflurane) requirement was found to be considerably lower. Also, the mean recovery time as indicated by the ability to vocalize following extubation was found to be significantly less. Hence, it can be recommended in laparoscopic cholecystectomy for maintaining cardiovascular system stability.

Keywords: Cardiovascular stability, Dexmedetomidine, Laparoscopic cholecystectomy, Post-op recovery.

How to cite this article: Dodeja H, Udaybhaskar V, Singam A. Effects of Dexmedetomidine Infusions on Hemodynamic Stability in Patients undergoing Laparoscopic Cholecystectomy. Int J Recent Surg Med Sci 2018;4(1):10-14.

Source of support: Nil

Conflict of interest: None

\footnotetext{
${ }^{1,2}$ Resident, ${ }^{3}$ Professor

${ }^{1-3}$ Department of Anesthesiology, Jawaharlal Nehru Medical College, Sawangi (Meghe), Wardha, Maharashtra, India

Corresponding Author: Amol Singam, Professor, Department of Anesthesiology, Jawaharlal Nehru Medical College, Sawangi (Meghe), Wardha Maharashtra, India, Phone: +9422538005 e-mail: amolsingam@rediffmail.com
}

\section{INTRODUCTION}

Hemodynamic stability during perioperative period is of paramount importance, as there are many patients who have a compromised cardiovascular status and are on medication. Critical events during perioperative period like induction, intubation, and surgical stimulus initiate metabolic response to trauma that needs to be considered and attended. In recent years, the laparoscopic surgeries which once upon a time were considered to cause least trauma are reported to have produced hemodynamic instability. ${ }^{1}$

The anesthesiologist's traditional approach to anesthesia for laparoscopic cholecystectomy has been the emphasis on maintaining hemodynamic stability by avoiding hypertension, hypotension, or tachycardia. The hemodynamic instability is persistent during the duration of PNP, namely, $\mathrm{CO}_{2}$ insufflations.

Volatile agents like isoflurane and sevoflurane ${ }^{1}$ have been used with limited success in maintaining hemodynamic stability as volatile agents decrease surgical stimulus-induced catecholamine secretion. Opioids have traditionally been used for blunting the perioperative stress response during general anesthesia. Although general anesthesia prevents hemodynamic instability by rendering patients insensate to pain of surgery, it is unable to completely eliminate the perioperative stress response. ${ }^{2}$

Laparoscopic surgeries require creation of $\mathrm{PNP}$ which is produced by insufflations of $\mathrm{CO}_{2}$ in the abdominal cavity by using automated flow-controlled carbon dioxide insufflator, which supplies gas till the required intraabdominal pressure is reached. Inflation pressure can be varied from 0 to $30 \mathrm{~mm} \mathrm{Hg}$, whereas the total gas flow volume can be set from 0 to $9.9 \mathrm{~L} / \mathrm{min}$.

Problems encountered during laparoscopic surgeries result from the combined effects of PNP with insufflations of carbon dioxide and patient positioning. ${ }^{3}$

After creation of PNP, intra-abdominal pressure increases along with the increase in circulating blood volume which is due to shifting of blood from the splanchnic capacitance blood vessel. Also, there is moderate increase in the intra-abdominal pressure which raises cardiac output and mean arterial pressure (MAP). ${ }^{4}$

As intra-abdominal pressures rises, circulating blood volume falls and venous return decreases, and consequently, there is a fall in cardiac output. This fall in cardiac 
output is troublesome in hypovolemic patients and patients receiving anesthetic agents with cardiac depressant effects. Laparoscopy induces significant hemodynamic changes and leads to increased systemic vascular resistance (SVR) and pulmonary vascular resistance, increase in MAP, reduction in stroke volume, cardiac output, and this mechanism is mechanically and humorally mediated. ${ }^{5}$

Alpha 2 agonists produce diverse responses, including analgesia, anxiolysis, sedation, and sympatholysis, each of which has been reported in the treatment of surgical and chronic pain patients and in panic disorders as well. ${ }^{4}$

Recently, the Food and Drug Administration registered two novel alpha 2-adrenergic agonists, clonidine and dexmedetomidine. ${ }^{6}$

Dexmedetomidine is seven to ten times more selective for alpha 2 receptors compared with clonidine, and has a shorter duration of action. Dexmedetomidine is considered full agonist at alpha 2 receptors as compared with clonidine, which is considered as a partial agonist. Dexmedetomidine attenuates the hemodynamic response to tracheal intubation, decreases plasma catecholamine concentration during anesthesia, and decreases perioperative requirements of inhaled anesthetics. ${ }^{7}$

As laparoscopic cholecystectomy is a routinely performed surgery, it is desirable to have a stable intraoperative hemodynamic status. Hence, this study attempted to find the beneficial effects of the alpha 2 agonist dexmedetomidine in maintaining the perioperative parameters like HR, SBP, DBP, MAP, etc.

\section{OBJECTIVE}

To study the efficacy of dexmedetomidine on cardiovascular system stability in patients undergoing laparoscopic cholecystectomy.

\section{MATERIALS AND METHODS}

This prospective study was conducted in the Anaesthesiology Department in Acharya Vinoba Bhave Rural Hospital in central India during the period from September 2015 to March 2017. The clearance for the study was obtained from the institutional ethics committee concerned. A written informed consent was obtained from each patient and each enrolled patient gave his/her written informed consent to participate.

Sample size was calculated with the formula:

$$
\begin{aligned}
& N=\left\{(4 \sigma 2)\left[Z\left(1-\left[\frac{\alpha}{2}\right]\right)\right]+Z(1-\beta) 2\right\} \\
& N=[(4 \sigma 2)(1-(\alpha / 2))+Z(1-\beta) 2]
\end{aligned}
$$

A sample size of $15(\mathrm{~N})$ patients each was randomly allocated into two groups using computerized randomization. Group P(placebogroup) received normalsalineand group D (dexmedetomidine group) received $0.4 \mu \mathrm{g} / \mathrm{kg} /$ hour of injection dexmedetomidine in $0.9 \%$ normal saline. Patients between ages of 20 and 50 years, within American Society of Anesthesiologists grades 1 and 2, undergoing elective laparoscopic cholecystectomy, under general anesthesia, were included in the study. Exclusions from the study included extremes of age groups, smokers, pregnant women, recent upper airway infections, and associated comorbid cardiac, respiratory, neurological, or metabolic complications.

A written informed consent was obtained from the patient. A thorough preanesthetic evaluation was performed by taking history and clinical examination and recorded on predesigned and pretested pro forma. For all patients, age, weight, height, SBP, DBP, and HR were recorded.

The study drug was provided, in dilutions of:

- Normal saline $0.9 \%-20 \mathrm{~mL}$

- Dexmedetomidine $20 \mathrm{~mL}(5 \mu \mathrm{g} / \mathrm{mL})$

Two intravenous (IV) lines were secured, one 20 G IV cannula in the right hand for the infusion and another 18 G IV cannula in the left hand for IV fluids and drug administration; $500 \mathrm{~mL}$ of crystalloids (Ringer Lactate) was started. Parameters, such as $\mathrm{HR}, \mathrm{MAP}$, and $\mathrm{SpO}_{2}$ using pulse oximeter were monitored before, during, and after the surgery. End tidal carbon dioxide was monitored intraoperatively and kept between 25 and $30 \mathrm{~mm} \mathrm{Hg}$.

The study drug was started 30 minutes before induction using an infusion pump at the rate of $0.08 \mathrm{~mL} / \mathrm{kg}$ body weight/hour. After shifting to operating room, monitors, electrocardiogram, noninvasive blood pressure amplifier, and pulse oximeter were attached. Patients were premedicated with injection midazolam $0.05 \mathrm{mg} / \mathrm{kg}$, injection fentanyl $1.5 \mu \mathrm{g} / \mathrm{kg}$ followed by preoxygenation for 3 minutes. Induced with propofol $2 \mathrm{mg} / \mathrm{kg}$, muscle relaxation was facilitated with injection vecuron $0.1 \mathrm{mg} / \mathrm{kg}$. Patients were intubated using an appropriate-sized endotracheal tube and maintained on $\mathrm{O}_{2}: \mathrm{N}_{2} \mathrm{O}(30: 70)$ and isoflurane $1 \%$ was started.

The MAP and HR were measured

- Preoperativlye (M1)

- 10 minutes after starting study drug infusion (M2)

- At induction (M3)

- After intubation (M4)

- Before PNP (M5)

- 10 minutes after PNP (M6)

- 20 minutes after PNP (M7)

- 30 minutes after PNP (M8)

Then, every 30 minutes till the end of surgery

- $\quad$ End of PNP (N1)

- After reversal (N2)

- Postoperative in recovery room (N3)

The study drug infusion was discontinued at the end of PNP. 


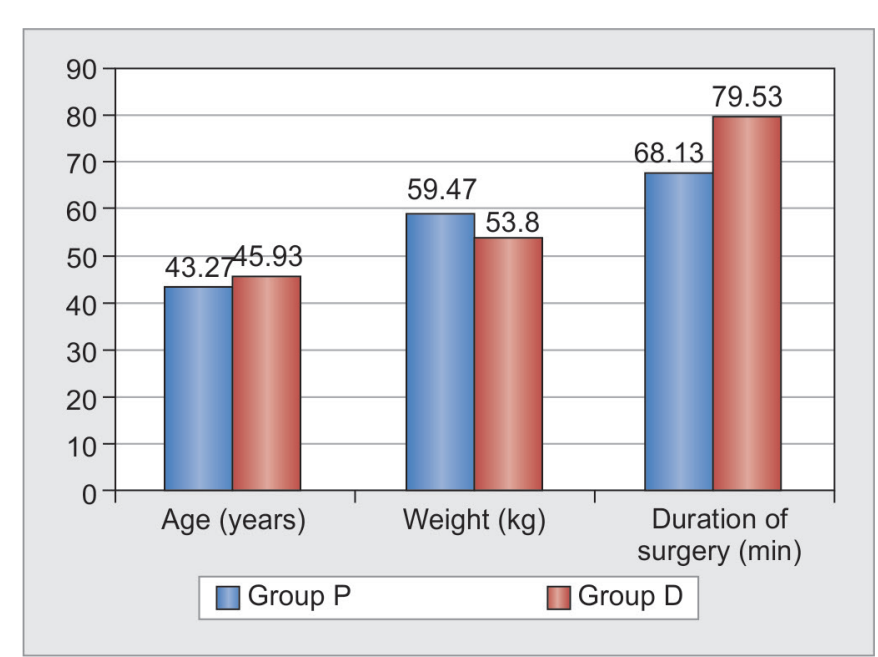

Graph 1: Patient characteristics. Average age in group P (placebo group) was 47.23 years and that in group $D$ (dexmedetomidine group) was 45.93 years. Average weight in group $\mathrm{P}$ (placebo group) was $59.47 \mathrm{~kg}$ and that in group $\mathrm{D}$ (dexmedetomidine group) was $53.80 \mathrm{~kg}$. Average duration of surgery in group P (placebo group) was 68.13 minutes and that in group $D$ (dexmedetomidine group) was 79.53 minutes

After surgery, patients were reversed with injection glycopyrrolate $0.01 \mathrm{mg} / \mathrm{kg}$ and injection neostigmine $0.05 \mathrm{mg} / \mathrm{kg}$. Patients were extubated and time to recovery was measured, recovery being defined as the time to vocalize after extubation. At the end of the study, the data were decoded and analysis was done as per the analysis plan.

\section{RESULTS}

The effects of dexmedetomidine were assessed for cardiovascular system stability on 30 patients undergoing elective laparoscopic cholecystectomy at Jawaharlal Nehru Medical College, Sawangi (Meghe) from 2015 to 2017. The results were noted.

Mean age (year), weight $(\mathrm{kg})$, and duration of surgery (min) are depicted in Graph 1.

\section{Recovery Time following Extubation}

Recovery time was assessed by ability to vocalize following the extubation. In group $\mathrm{P}$, mean time interval to vocalize was $6.8 \pm 2.40$ minutes, whereas in group $\mathrm{D}$, it was $3.46 \pm 2.03$, which was statically significant ( $p$-value $<0.0001)$.

\section{Isoflurane Requirement (Number of Patients requiring 1 to $1.5 \%$ Isoflurane)}

All the patients (15) in group P required 1 to $1.5 \%$ isoflurane during the intraoperative period, whereas $5(33.33 \%)$ patients in group D required 1 to $1.5 \%$ isoflurane during the intraoperative period. Hence, the requirement of the inhalation agent was significantly reduced in group D (dexmedetomidine group) (Graphs 2 and 3).

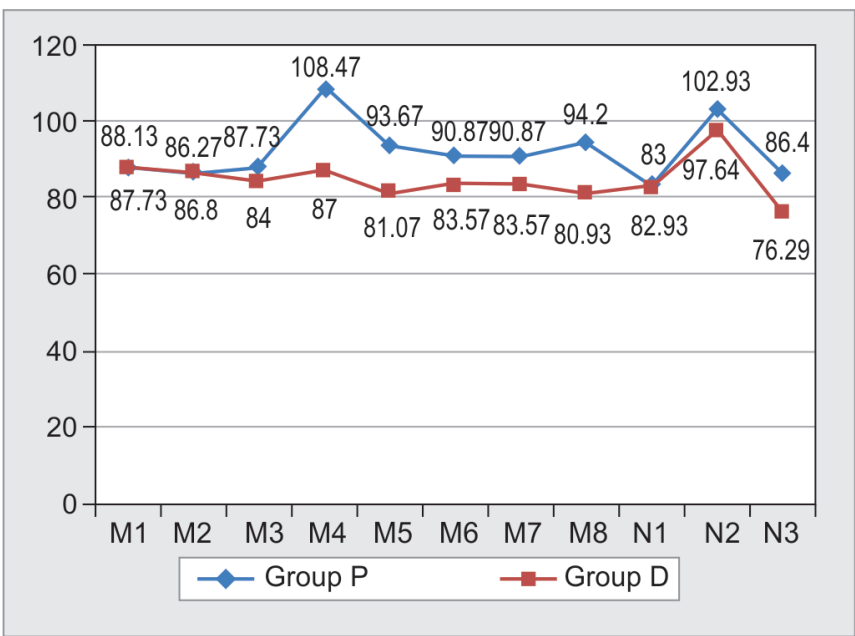

Graph 2: Heart rate. Heart rate in group $P$ (placebo group) increased significantly after intubation (M4), before PNP (M5), 10 minutes after PNP (M6), 20 minutes after PNP (M7), 30 minutes after PNP (M8), and postoperatively in recovery (N3) when compared with group $D$ (dexmedetomidine group)

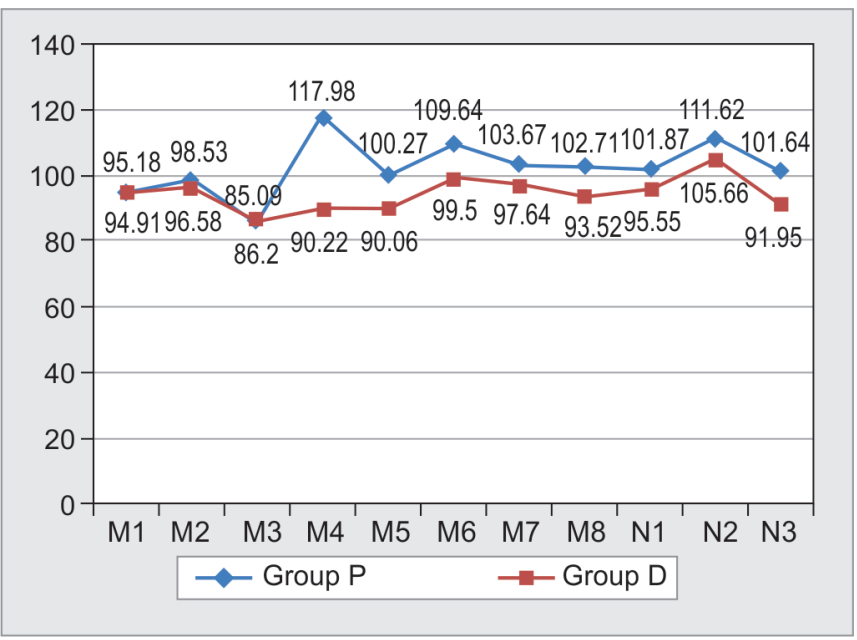

Graph 3: Mean arterial pressures. The MAP in group P (placebo group) was significantly higher after intubation (M4) and postoperatively in recovery $(N 3)(p \leq 0.05)$ compared with group $D$ (dexmedetomidine group)

\section{Use of Atropine (0.6 mg IV)}

In this study, atropine requirement was found in $1(6.67 \%)$ patient in group D (dexmedetomidine group) due to bradycardia.

\section{DISCUSSION}

Patients undergoing laparoscopic cholecystectomy are prone to intraoperative hypotension ${ }^{5}$ and tachycardia. There is increase in SVR and it is associated with a decrease in cardiac index and metabolic changes. In this study, we have observed the efficacy of dexmedetomidine on the hemodynamic stability in patients undergoing laparoscopic cholecystectomy. 
There was a significant difference in the HR and MAP in group $\mathrm{P}$ (placebo group) and group D (dexmedetomidine group) after laryngoscopy and intubation (M4), and postoperative in recovery (N3).

In a study, ${ }^{7}$ clonidine $4 \mu \mathrm{g} / \mathrm{kg}$ and dexmedetomidine $2.5 \mu \mathrm{g} / \mathrm{kg}$ were given 40 to 50 minutes before the anticipated induction of anesthesia and it was found that HR and MAP were found to be lower in clonidine and dexmedetomidine group when compared with the placebo group. We noticed in our study that HR and MAP were significantly lower in group D (dexmedetomidine group) than in group P (placebo group).

We found that in group D (dexmedetomidine group), the HR and MAP remained similar to the preoperative value during the PNP, thus indicating the hemodynamic stability during PNP with dexmedetomidine when compared with group $\mathrm{P}$ (placebo group). Significant difference was found during laryngoscopy and intubation (M4). Similar results were also found by studies done by others. ${ }^{8,9}$

A study ${ }^{10}$ compared three infusion doses of dexmedetomidine, $0.2,0.4$, and $0.8 \mu \mathrm{g} / \mathrm{kg} / \mathrm{hour}$, with saline in morbidly obese patients undergoing laparoscopic bariatric surgery. Mean arterial blood pressure values were maintained within $\pm 25 \%$ of the preinduction baseline values by varying the inspired desflurane concentration. It was found that intraoperative hemodynamic values were similar in the four groups, arterial blood pressure values were significantly reduced in the dexmedetomidine $0.2,0.4$, and 0.8 groups compared with the control group on admission to the postanesthesia care unit (PACU) $(\mathrm{p}<0.05)$. In our study also, the MAP in the dexmedetomidine group was significantly less in PACU $(\mathrm{p}<0.05)$.

A study ${ }^{11}$ showed the effects of dexmedetomidine infusion $(0.2 \mu \mathrm{g} / \mathrm{kg} /$ hour $)$ for hemodynamic stability in patients undergoing laparoscopic cholecystectomy and found that MAP and HR in the dexmedetomidine group were significantly less after intubation and throughout the period of PNP. We found similar result only after intubation, but there was no statistically significant difference during the period of PNP between the dexmedetomidine and saline groups. It appears that the dexmedetomidine group maintained MAP and HR throughout the PNP without additional isoflurane requirement, whereas in the saline group, higher MAC values of isoflurane were required to control $20 \%$ rise above preoperative values.

There was a reduction in isoflurane requirement in group D (dexmedetomidine group) as compared with group P (placebo group); hence, mean recovery time as indicated by ability to vocalize following extubation was found to be significantly less in group D (dexmedetomidine group). Isoflurane was used only in 5 of 15 patients $(33 \%)$ in the dexmedetomidine group and in all the patients $(100 \%)$ in the saline group. A reduction of isoflurane requirement was observed in our study as lower MAC (minimum alveolar concentration) and inhalational agent at lower dial concentration was required. The patients required significantly lower concentrations of isoflurane in the dexmedetomidine group. Our findings are in accordance with other studies, in which there was decrease in MAC and inhalational agent requirement. ${ }^{12,13}$

There was delayed recovery in the placebo group compared with the dexmedetomidine group which appears to be due to higher consumption of isoflurane.

Atropine was used in 1 out of 15 patients $(6.67 \%$ patients) in the dexmedetomidine group, when the HR decreased to less than 50 per minute. Previous studies ${ }^{14}$ have demonstrated bradycardia associated with dexmedetomidine administration.

\section{CONCLUSION}

The drug dexmedetomidine maintained cardiovascular stability during laparoscopic cholecystectomy. It was effective in maintaining perioperative cardiovascular system stability during laparoscopic cholecystectomy. Hence, it can be recommended for maintaining cardiovascular system stability during laparoscopic cholecystectomy.

\section{REFERENCES}

1. Aono H, Takeda A, Tarver SD, Goto H. Stress responses in three different anesthetic techniques for carbon dioxide laparoscopic cholecystectomy. J Clin Anesth 1998 Nov;10(7): 546-550.

2. Luchetti M,Palomba R,Sica G,Massa G, Tufano R. Effectiveness and safety of combined epidural and general anesthesia for laparoscopic cholecystectomy. Reg Anesth 1996 Sep-Oct; 21(5):465-469.

3. Glick, D. Miller's anesthesia. 7th ed. New York: Churchill Livingstone; 2010.

4. Yildiz M, Tavlan A, Tuncer S, Reisli R, Yosunkaya A, Otelcioglu S. Effect of dexmedetomidine on haemodynamic responses to laryngoscopy and intubation. Drugs R D 2006 Jan;7(1):43-52.

5. Joris J, Noirot D, Legrand M, Jacquet NJ, Lamy M. Hemodynamic changes during laparoscopic cholecystectomy. Anesth Analg 1993 May;76(5):1067-1072.

6. Kamibayashi T, Maze M. Clinical uses of $\alpha 2$-adrenergic agonists. Anesthesiology 2000 Nov;93(5):1345-1349.

7. Taittonen MT, Kirvelä OA, Aantaa R, Kanto JH. Effect of clonidine and dexmedetomidine premedication on perioperative oxygen consumption and haemodynamic state. Br J Anaesth 1997 Apr;78(4):400-406.

8. Vora KS, Baranda U, Shah VR, Modi M, Parikh GP, Butala BP. The effects of dexmedetomidine on attenuation of hemodynamic changes and there effects as adjuvant in anesthesia during laparoscopic surgeries. Saudi J Anaesth 2015 Oct-Dec; 9(4):386-392.

9. Singh V, Yadav RR. Effect of dexmedetomidine on hemodynamic stability during laparoscopic surgeries: a randomized 
double-blind controlled trial. Int J Med Sci Public Health 2016;5(12):2555.

10. Tufanogullari B, White PF, Peixoto MP, Kianpour D, Lacour T, Griffin J, Skrivanek G, Macaluso A, Shah M, Provost DA. Dexmedetomidine infusion during laparoscopic bariatric surgery: the effect on recovery outcome variables. Anaesth Analg 2008 Jun;106(6):1741-1748.

11. Bhattacharjee DP, Nayek SK, Dawn S, Bandopadhyay G, Gupta K. Effects of dexmedetomidine on haemodynamics in patients undergoing laparoscopic cholecystectomy-a comparative study. J Anaesth Clin Pharmacol 2010 Jan;26(1):45-48.
12. Aantaa R Jaakola ML, Kallio A, Kanto J. Reduction of the minimum alveolar concentration of isoflurane by dexmedetomidine. Anesthesiology 1997 May;86(5):1055-1060.

13. Singh S, Arora K. Effect of oral clonidine premedication on perioperative haemodynamic response and postoperative analgesic requirement for patients undergoing laparoscopic cholecystectomy. Indian J Anaesth 2011 Jan-Feb;55(1):26-30.

14. Aho M, Erkola OA, Scheinin H, Lehtinen AM, Korttila KT. Effect of intravenously administered dexmedetomidine on pain after laparoscopic tubal ligation. Anesth Analg 1991 Aug;73(2):112-118. 